\title{
Calculation and analysis of the wave load of jack-up offshore wind turbine installation vessel during dynamic positioning
}

\author{
Zhenqiu Yao ${ }^{a}$, Yun Shen ${ }^{b}$, Xin $\mathrm{Gu}^{\mathrm{c}}$ and Linxin $\mathrm{Bu}^{\mathrm{d}}$
}

School of Naval Architecture and Ocean Engineering. Jiangsu University of Science and Technology, Zhenjiang, China

ayaozq_just@126.com, byzshenyun@126.com, c1010224404@qq.com, d493961936@qq.cpm

Keywords: Jack-up offshore wind turbine installation platform, Near Field Integral Theory, Wave loads.

\begin{abstract}
The jacking-up offshore wind turbine installation vessel will under complex ocean condition during its sailing, positioning and working, and the wave loads are needed to be calculated and analyzed as the most complex loads. This paper adopts Near Field Integral Theory which based on integral surface, using ANSYS software to establish the precise geometric model, and then calculate wave loads and motion response transfer function through the AQWA modules for wind power installation vessel in three degrees of freedom and different angles to the flow direction. Meanwhile, the calculation shows that wave direction has a greater impact on the motion response of jacking-up offshore wind turbine installation vessel.
\end{abstract}

\section{Preface}

The third generation of self-propelled jack-up offshore wind turbine installation vessel has a function of transport platform and operation platform, and it has the vehicle navigation, hull comprehensive functions such as lifting work, strong ability to resist the wind and waves, and high efficiency, which has become the mainstream of sea fan installed equipment. Because of the work environment of sea wind turbines, when wind power installation vessel operates in rough large offshore wind farm area, it needs a stable working environment, so the vessel equips dynamic positioning system. The ship's hull is usually relatively flat, and equips heavy crane as well as a number of root pile legs and boots, and the ship can also load and transport several powerful wind power equipment. Because of the large wind area and high center of gravity, dynamic positioning system must resist the complex wind, wave and current load which the hull suffered. In the total environmental loads which the ship suffered, movement of the target ship in the direction of the three degrees of freedom caused by waves should not be ignored, and especially the slow drift caused by the second-order wave force which is the most serious, so wave load calculation is very necessary for jack-up offshore wind turbine installation vessel.

This paper takes a medium-sized jack-up offshore wind turbine installation vessel as the research object, and uses the application analysis software ANSYS ${ }^{[1]}$ which is based on the Near Field Integral Theory of the object surface integral in consideration of the external environment and the characteristics of ship type to calculate and analyze the wave load on the ship hull in the process of dynamic positioning, as well as the wave response of this ship.

\section{The calculation method of load and motion response}

St. Denis and Pierson theories ${ }^{[2-3]}$ suggest that the motion response of floater on the waves is determined by the regular wave response (RAO) and the wave spectrum. The motion response of the wind power installation of ship can be calculated through RAO and wave spectrum analysis method of statistical forecast.

Calculation of ship motion and load in regular wave. The calculation of ship motion and load in regular wave always use the three-dimensional source theory ${ }^{[4]}$, and its basic assumption is:

The ship motion and wave is slightly linear;

The water is no viscous fluid; 
Concern the effects of diffraction and radiation;

Establish the whole hull frequency equation of motion:

$\left[-\omega^{2}(M+A(\omega))+i \omega\left(B(\omega)_{p}+B_{v}\right)+C\right] x(\omega, \beta)=F(\omega, \beta)$

In the formula: $\omega$ - angular frequency of the incident wave; $\beta$ - spread direction of the incident wave ; M- inertia matrix of the floating body ; $\mathrm{A}(\omega)$-added mass matrix related to the frequency ; $\mathrm{B}(\omega)_{p}$-radiation damping matrix related to the frequency; $B_{v}$ - linear damping matrices related to the viscosity of fluid; C- stiffness matrix of hydrostatic reply; $x(\omega, \beta)$ - movement matrix of floating body; $F(\omega, \beta)$ - matrix of wave exciting force; consist of the wave exciting force effect on the large scale component, the inertial force acting in small scale component and drag force.

It uses potential flow theory to solve three-dimensional hydrodynamic coefficient matrix [A] and [B], and ignores the fluid viscous effect ${ }^{[2]}$.

The effect of the second-order wave forces ${ }^{[5-6]}$. In the total environmental loads which the ship suffered, movement of the target ship in the direction of the three degrees of freedom caused by waves should not be ignored. During the operation process of wind power installation vessel positioning, it may cause the condition that the same as the wave frequency which may result in strenuous movement on the direction of the degrees of freedom. What's more, the effects of slow drift caused the second-order wave force is more seriously. It can cause the deviation of the average position of ship motion and lead to slow drift motion of the ship. This has an effect on the ship with the dynamic positioning system, and makes the ship away from the given position which results in the failure of positioning. So it is necessary to calculate the force during the positioning system design.

The calculation theories of second-order wave force includes the Near Field Integral Theory which is based on the object surface integral ${ }^{[7]}$, the Far Field Theory which is based on the theory of momentum and energy conservation and the Midfielder Integral Theory. Among them Pinkster's Near Field Integral Theory describes the formation mechanism of the second order wave forces, and theoretical significance of each component of the theory. Meanwhile the calculations are much more accurate, although part of the problems (Solution of the second order potential) have not been fully resolved, it has widely used in numerical calculation in practical engineering. The Near Field Integral Theory will be used in the calculation of the second order wave force in this paper.

RAO and the response spectrum. $\mathrm{RAO}^{[8]}$ is the response of the floating body under unit amplitude simple harmonic wave. The function changed with time which is under the action of a simple harmonic wave the response can be written as:

$$
R(\omega, \beta, t)=A \cdot \operatorname{Re}\left[|H(\omega, \beta)| \cdot e^{i(\omega t+\varphi)}\right]
$$

$H(\omega, \beta)$ Is the RAO.

When we finish the calculation of $H(\omega)$, the response spectrum ${ }^{[10]}$ of structure can be obtained: depend on the spectrum $S_{\eta}(\omega)$

$$
S_{R}(\omega)=S_{\eta}(\omega) \cdot|H(\omega)|^{2}
$$

The analysis calculation of motion response relied on the AQWA module which is based on the theory of near field integral in ANSYS software. The module can be used to analyze any fixed or floating structures and zero velocity wave interaction in the finite and the infinite depth.

\section{Calculation of model}

The research object of this article is a self-propelled jack-up offshore wind turbine installation vessel which is working in the wind farms of Jiangsu coastal. The vessel is a new type of ship which includes hull, forecastle, crane, hydraulic pile legs and so on and has the functions of self-propulsion, transportation, self-raise platform and lifting, and meanwhile it has a migration speed of $10 \mathrm{KN}^{[11]}$. The maximum working depth of this ship is $25 \mathrm{~m}$, and it equips DP-2 dynamic positioning system. The ship's layout diagram is shown in figure 1, the main parameters are shown in table 1. 


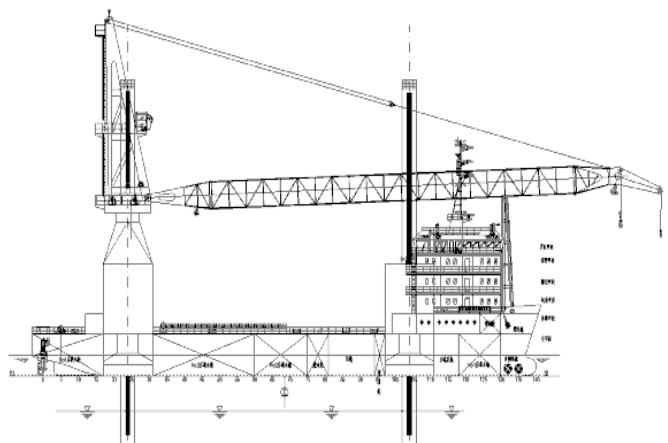

Figure 1 Layout diagram

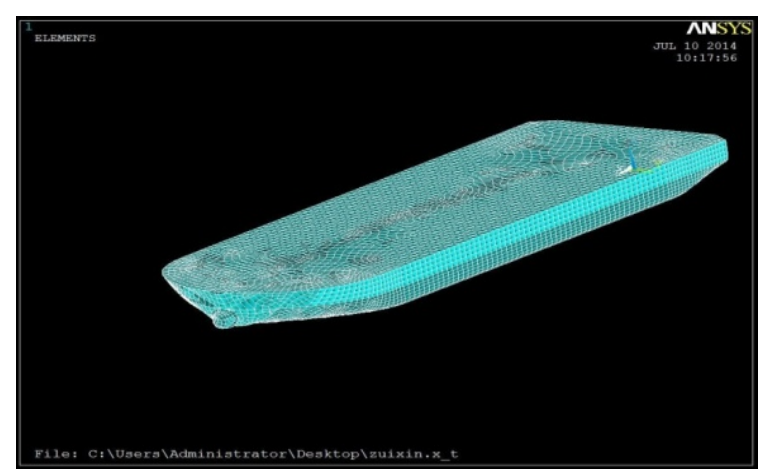

Figure 2 Elements model

Table 1 Main parameters

\begin{tabular}{cccccc}
\hline LOA & LPP & B & D & d & CB \\
\hline $86.69 \mathrm{~m}$ & 82.20 & $30.00 \mathrm{~m}$ & $6.00 \mathrm{~m}$ & $3.00 \mathrm{~m}$ & 0.85 \\
\hline
\end{tabular}

We use Solid works to establish the solid model of this ship, then imported it into ANSYS for elements partition. We divided by shell 181, and the length of elements below waterline is $500 \mathrm{~mm}$, the length above waterline is $750 \mathrm{~mm}$. The number of nodes is 11392 and the number of elements is 11413. We use AQWA to calculate the hydrodynamic force. Coordinate system $\mathrm{x}$ direction pointing to the bow, Y direction refers to port, $\mathrm{Z}$ direction pointing up. Origin of coordinates in the baseline, 0 station number. Elements model is shown in figure 2.

\section{The example analysis}

In this paper, we use the method of Near Field Formula and rely on the AQWA software to calculation. The main analysis module used in calculation is AQWA-LINE. The module is mainly used for calculation of floating body structure in regular wave response by using the typical Green's function method to calculate the wave force around the floating body structure of arbitrary shape caused by wave radiation/ diffraction, added mass, radiation damping and freedom movement of floating body on the direction of six degrees. Also it considers the structure of the floating body structure and the nearby solid hydrodynamic interactions at the same time, able to handle the shallow water effect condition (Wave forces acting on the high-order item should be considered).

By the content which is described earlier we can know that the ship form and the arrangement of propulsion system are symmetrical relative to the overall structure of longitudinal profile. So when we calculate the second order wave force, we just need to consider the change of the external load direction in the range 0 to 180 , and the wind speed of $14 \mathrm{~m} / \mathrm{s}$, significant wave height is $2.5 \mathrm{~m}$, due to space limitations in this list only the load direction every $15^{\circ}$ calculation, finally take the wave frequency $\omega\left(\omega=\frac{T}{2 \pi}\right.$,T for the wave period ) range from $0.1 \mathrm{rad} / \mathrm{s}$ to $2.3 \mathrm{rad} / \mathrm{s}$, every 0.05 to get a point, connect these points in turn to get amplitude response operator and second-order wave force diagram.

We calculate the RAO of second order wave drift force slow under the condition of different wave direction angles and different frequencies in surge, sway, yaw three degrees of freedom by AQWA.

The graphs below show the RAO in three degrees of freedom of the wind power installation vessel under 3 m draft.

It shows that surge motion amplitude of the hull decreases continuously with the increase of wave frequency by analyzing the results above. The amplitude reaches maximum and minimum at the wave angle of $180^{\circ}$ (following sea) and $90^{\circ}$ (Beam Sea). In the same way, the swaying amplitude decreases too when wave frequency goes up. The range of motion reaches maximum at the wave angel of $90^{\circ}$ and reaches minimum at $0^{\circ}$ or $180^{\circ}$. The maximum response operator RAO shows up when the wave frequency is $0.1 \mathrm{rad} / \mathrm{s}$. The largest gap of the hull rocking motion amplitude is between the wave angel of $45^{\circ}$ and $135^{\circ}$. The amplitude reaches maximum at the wave frequency of 0.1 and reaches minimum at the wave angel of $180^{\circ}$ or $0^{\circ}$. 


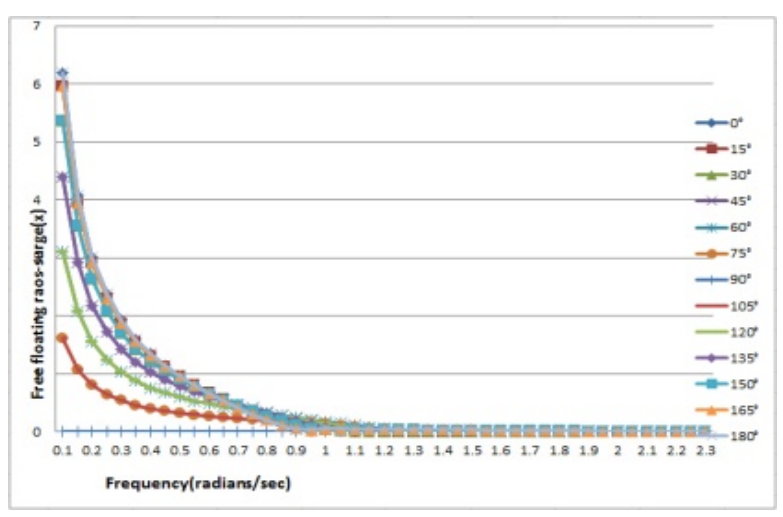

Figure 3 Surge RAO

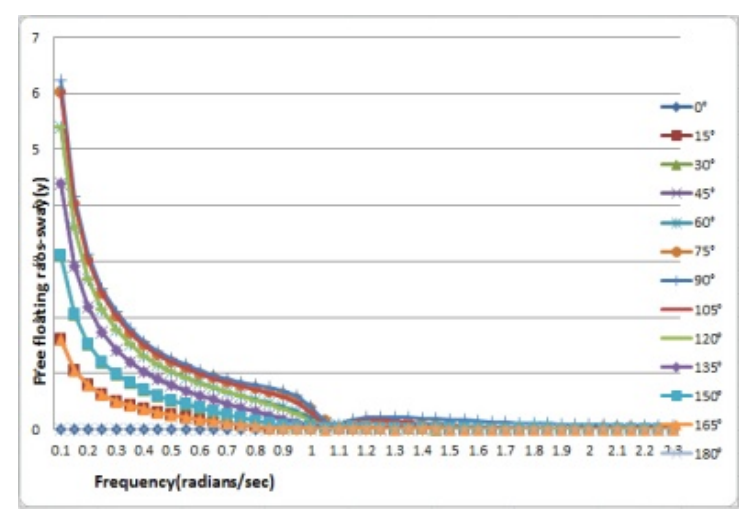

Figure 4 Sway RAO

The condition of the second-order wave drift force of surge, sway and yaw at different wave angel and frequency is showed in figure $6,7,8$.

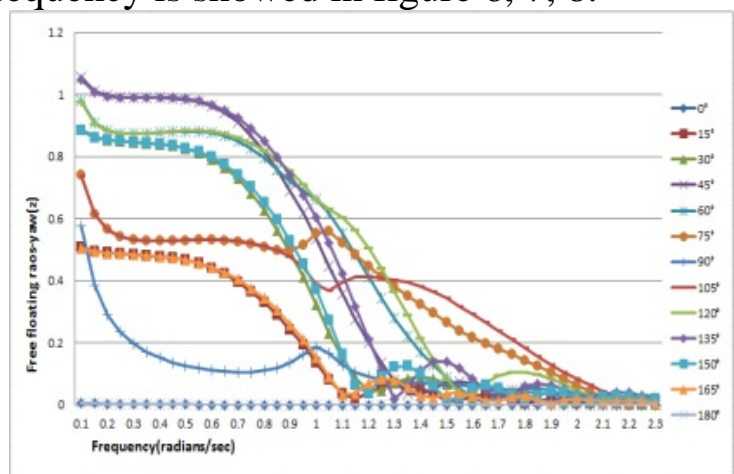

Figure 5 Yaw RAO

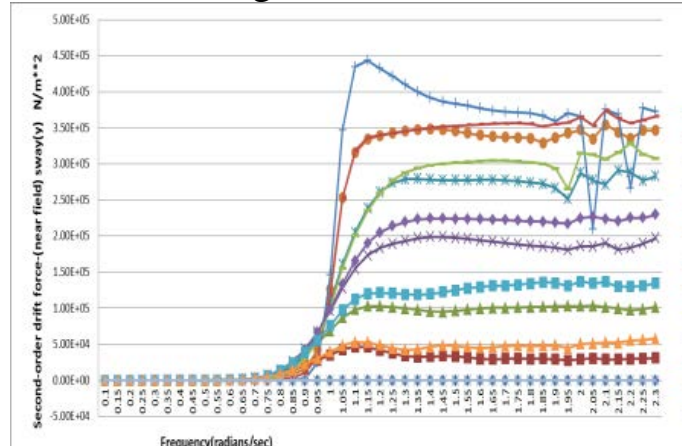

Frequency(radians/sec)

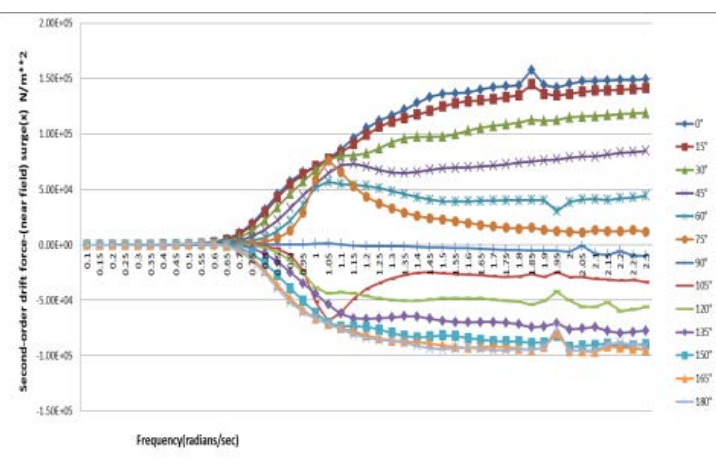

Figure 6 Surge second-order wave drift force

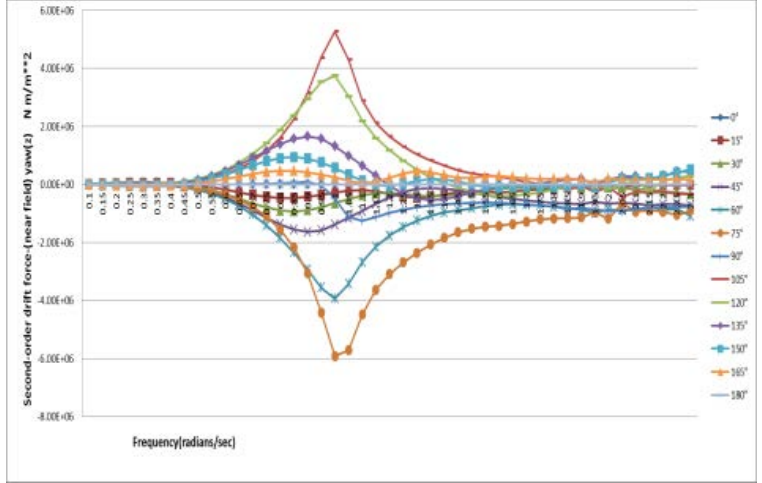

Figure 8 Yaw drift force wave drift force

Figure 7 Sway second-order wave drift force

Surge second-order drift force increase with the increase of wave frequency, the positive direction second- order drift force reaches maximum of $1.49 \mathrm{E}+05$ at $0^{\circ}$ between $0^{\circ}$ and $90^{\circ}$, the negative direction second-order drift force reaches maximum of $-9.12 \mathrm{E}+04$ at $180^{\circ}$ between $90^{\circ}$ and $180^{\circ}$, both of these two condition's frequency are $2.3 \mathrm{rad} / \mathrm{s}$. The overall trend of sway second-order drift force is increasing with the increase of wave frequency, the maximum is $4.43 \mathrm{E}+05$ when the wave angel is $90^{\circ}$ and the frequency is $1.15 \mathrm{rad} / \mathrm{s}$, the minimum is nearly 0 when the wave angle is $0^{\circ}$ or $180^{\circ}$.The yaw second-order drift torque reaches maximum with the wave frequency increasing, and then decrease to a stable condition. The maximum is $5.91 \mathrm{E}+06$ when the wave frequency is $1 \mathrm{rad} / \mathrm{s}$ and the wave angel is $75^{\circ}$. When wave angel increases to $105^{\circ}$ and the frequency is $1 \mathrm{rad} / \mathrm{s}$, the max torque is $5.26 \mathrm{E}+06$.

\section{Conclusion}

According to near field integral theory, the paper uses the modular AQWA-LINE of ANSYS to finish the calculation of three degrees of freedom motion of jack-up offshore wind turbine installation vessel and obtain motion response parameters of response operator and two order drift force with different wave angel and wave frequency: 
The motion response of the jack-up offshore wind turbine installation vessel is greatly affected by wave angel, surge motion reaches maximum at $180^{\circ}$, sway at $90^{\circ}$ and yaw at frequency of 1 , angel $45^{\circ}$, $135^{\circ}$. And the surge and sway trends to decrease with the increasing of wave frequency.

The second-order wave drift force is affected by wave angel too, the surge second-order drift force reaches maximum at $0^{\circ}$ and $180^{\circ}$, the sway at $90^{\circ}$, and the yaw second-order drift torque at frequency 1 with angel $75^{\circ}$ and $105^{\circ}$. Surge and sway second-order drift force increase with the increase of wave frequency, the yaw second-order drift torque reaches maximum with the wave frequency increasing, and then decrease to a stable condition.

Under the condition of oblique wave, longitudinal shear and the horizontal axis torque response reach maximum, it affects main deck so the demand of its torsional strength is high. Under the transverse wave condition, the horizontal separation force affects mostly, so the strength of main deck and the connection of the hull requires higher standard. Under the head sea condition, the vertical wave bending moment is larger, wave peak and trough is at the middle of the ship hull, so the overall flexural capacity of ship should be enough.

\section{References}

[1] Wang Guo-Qiang. Numerical simulation of the practical project and practice on the ANSYS[M]. Xi 'an: Northwestern polytechnical university press, 1999

[2] Zhang Hai-bin, Cheng Wei-Jie. Hydrodynamic analysis for U-style semi-submersible hoisting and pipe-laying vessel [J]. Marine Design \&Research Institute of China, 2010(11), 28(04):41

[3] St Denis M, Pierson W J Jr. On the motions of ship in confused sea[J]. Trans. SNAME, 1953, 61: p. 280-358

[4] Feng Tie-cheng, Zhu Wen-wei, Gu Shu-hua. The ship maneuvering and oscillation[M],1989

[5] MauroH. The drift of a body floating in wave, J. of Ship Research,1960.

[6] Newman J N. The drift force and moment on ships in waves,J. of Ship Research,Vol.4,March 1967.

[7] Pinkster J A. Low frequency second order wave forces on vessels moored at sea. Proc. 11 thsymp. On naval hydrodynamics. Univ. coll. London. 1976,603-615.

[8] Xiaozhi Wang; Torgeir Moan Stochastic and deterministic combination of still water and wave bending moment on a containership 1996(09)

[9] O.M.Faltinsen. Ships and Marine engineering environmental load[M].Shanghai: Shanghai Jiaotong university press, 2008:100-101

[10] YAO Zhen-qiu, MA Yi-meng, HAN Qiang, YAN Zhou-guang, Whole hull's structural strength analysis of offshore wind turbine installation vessel [J]. Journal of Ship Mechanics, 2012(02), 16(1-2)

[11] Zhang Tai-ji, Wang Zhang-tang, Development of new ship type-maritime wind turbine installation vessel [J]. Ship\&Boat, 2009,5

[12] Zhang Wei, Hydrodynamic analysis for a deep-water semi-submersible drilling platform [D]. Shanghai Jiaotong university press, 2006

[13] Liu Ying-Zhong, Miu Guo-Ping, The motion theory of the ship on the wave. .Shanghai: Shanghai Jiaotong university press, 1984

[14] Dai Yang-shan, Shen Jin-wei, Song Jing-zheng. Ship wave loads. National defence industry press, 2007

[15] WANG Lei, WANG Xian-fu, YANG Jian-min. Experimental research on second-order wave drift force on a dynamically positioned ship, The ocean engineering, 24(3),1-5

[16] Huang Hai-bo. Research on design of DP thruster system of wind farm installation vessel. Shanghai Jiaotong university, 2012. 